\title{
Caracterización florística de las especies de aprovechamiento apícola en el complejo volcánico "Pilas el Hoyo"
}

\section{Floristic characterization of the species of apicultural use in the volcanic complex "Pillas el Hoyo"}

\author{
Aguilar Cabrera, Ángel Benito; Aker-Narvaez, Carlos; Flores-Pacheco, \\ Santiago A.; Editor Academico Prof. Angel Sol-Sánchez
}

\author{
Ángel Benito Aguilar Cabrera \\ 110194aguilarangel@gmail.com \\ Universidad Nacional Autónoma de Nicaragua, León, \\ Nicaragua \\ Carlos Aker-Narvaez \\ carlos.aker@ev.unanleon.edu.ni \\ Universidad Nacional Autónoma de Nicaragua, León., \\ Nicaragua \\ Santiago A. Flores-Pacheco \\ santiago.pacheco@ev.unanleon.edu.ni \\ Universidad Nacional Autónoma de Nicaragua, León., \\ Nicaragua \\ Editor Academico Prof. Angel Sol-Sánchez \\ Colegio de Postgraduados Mexico, México
}

Revista Iberoamericana de Bioeconomía y Cambio Climático

Universidad Nacional Autónoma de Nicaragua, León, Nicaragua ISSN-e: 2410-7980

Periodicidad: Semestral

vol. 5, núm. 9, 2019

czuniga@ev.unanleon.edu.ni

Recepción: 11 Mayo 2019

Aprobación: 26 Junio 2019

URL: http://portal.amelica.org/ameli

jatsRepo/394/3941756007/index.htm

DOI: https://doi.org/10.5377/ribcc.v5i9.7952

Autor de correspondencia: 110194aguilarangel@gmail.com
Resumen: El estudio se llevó a cabo en el área protegida “Complejo Volcánico Cerro Negro - Pilas - El Hoyo", ubicada a $25 \mathrm{~km}$ al este de la ciudad de León. El objetivo de este estudio fue caracterizar las especies melíferas de la zona. Mediante la presente investigación los apicultores tendrán mayor conocimiento sobre las especies melíferas y su disponibilidad durante el año. Se eligieron 7 apiarios activos distribuidos entre la comunidad de Miramar y la comarca de los Terreros, donde se realizaron 4 muestreos semanales por apiario usando transectos de $20 \mathrm{~m}$ x $50 \mathrm{~m}$, tomando como punto de partida los apiarios para identificar y contar el número de especies de aprovechamiento apícola. Los datos obtenidos fueron exportados a Excel. Se identificaron 89 especies pertenecientes a 39 familias, dividas en: 59 especies de árboles, 15 especies de arbustos y 15 especies de hierbas. Las familias con mayor abundancia en árboles fueron las Bignoniáceae, Myrtaceae, Fabácea, Ebenácea y Boraginácea; en arbustos son la Verbenácea, Boraginaceae, Fabaceae, Apocynaceae y Solanaceae; en hierbas son la Plantaginacea, Malvaceae, Sterculiaceae, Lamiaceae y Convolvulacea. El uso de la tierra en los apiarios muestra un $53 \%$ de bosque silvestre, $12 \%$ de barbecho, $8 \%$ de maní, $12 \%$ entre maíz y sorgo, $9 \%$ entre eucalipto, frijol blanco y yuca, $4 \%$ entre potreros y caña, $2 \%$ entre pastizales y ajonjolí. Existe una mayor floración entre los meses de Diciembre a Mayo. De Junio a Noviembre hay escasez de floración, este fenómeno se trata de compensar con especies de floración prolongada y cultivos.

Palabras clave: Especies melíferas, Apiario, Bignoniáceae, Plantaginacea, Convolvulacea.

Abstract: The study was carried out in the protected area "Volcanic Complex Cerro Negro - Pilas - El Hoyo", located at $25 \mathrm{~km}$ east of the city of León. The objective of this study was to characterize the honey species in the area. Through this research, beekeepers will have greater knowledge about honey species and their availability during the year. Seven active apiaries distributed between the community of Miramar and the district of Terreros were chosen, where 4 weekly samplings per apiary were carried out using transects of $20 \mathrm{~m} \times 50 \mathrm{~m}$, taking as starting point the apiaries to identify and count the number of species of beekeeping. The data obtained were exported to Excel. We identified 89 species belonging to 39 families, divided into: 59 tree species, 15 shrub species and 15 herbal species. The 
families with greater abundance in trees were the Bignoniáceae, Myrtaceae, Fabácea, Ebenaceae and Boraginaceae; in shrubs are Verbenaceae, Boraginaceae, Fabaceae, Apocynaceae and Solanaceae; in herbs are Plantaginacea, Malvaceae, Sterculiaceae, Lamiaceae and Convolvulacea. The use of the land in the apiaries shows $53 \%$ of wild forest, $12 \%$ of fallow, $8 \%$ of peanuts, $12 \%$ between corn and sorghum, 9\% between eucalyptus, white beans and cassava, $4 \%$ between paddocks and cane, $2 \%$ between pasture and sesame. There is a greater flowering between the months of December to May. From June to November there is a shortage of flowering, this phenomenon is to compensate with species of prolonged flowering and crops.

Keywords: Honey species, Apiary, Bignoniáceae, Plantaginacea, Convolvulacea.

\section{INTRODUCCIÓN}

La ciudad de León se encuentra ubicado al oeste, en la región del pacífico, cuya vegetación original correspondía al bosque seco. Es importante conocer el bosque seco para entender la flora arbórea y arbustiva. Así, se debe mencionar que en estos bosques secos se cambió extensivamente el uso de la tierra y se destinaron a las actividades agrícolas desde hace tiempo. Esto ha generado una dramática alteración del ecosistema y se estima que menos del 1\% de la vegetación original persiste y no se encuentra en estado natural. (Paguaga y Soto.2010).

Según Paguaga y Soto, 2010, el bosque seco se puede describir como un bosque con un solo dosel bajo, generalmente de hasta 25 metros de altura. Acá se pueden encontrar arbustos como: el papamiel (Combretum farinosum, Combretaceae), que es una especie común y las epífitas que son poco diversas, Brassavola nodosa (Orchidaceae) y Tillandsia schiedeana (Bromeliaceae) son las más fáciles de encontrar. Las especies de árboles más comunes son: el ron-rón (Astronium graveolens, Anacardiaceae), el jiñocuabo (Bursera simaruba, Burseraceae) el madroño (Calycophyllum candidissimum, Rubiaceae), la ceiba (Ceiba pentandra, Bombacaceae) el guácimo molenillo (Luehea candida, Tiliaceae) y la mora (Maclura tinctoria, Moraceae), entre otras. Además, en lugares donde tienen acceso al agua subterránea es común observar genízaro (Albizia saman, Fabaceae), carao (Cassia grandis, Fabaceae), guanacaste (Enterolobium cyclocarpum, Fabaceae) y guapinol (Hymenaea courbaril, Fabaceae).

Formando parte del bosque seco se encuentran las Sabanas de Jícaro, que se encuentran principalmente en suelos rocosos poco profundos. Aquí las especies de árboles y arbustos son pocas, siendo las principales el jícaro sabanero (Crescentia alata, Bignoniaceae), acompañada por el cornizuelo (Acacia collinsii, Fabaceae), el nancite (Byrsonima crassifolia, Malpighiaceae) y el nacascolo (Caesalpinia coriaria, Fabaceae), entre otras. Son abundantes los pastos y las hierbas. Los pastos están representados en su mayoría por la familia Poaceae, cuyas especies comunes son Aristida ternipes, Bouteloua alamosana y Oplismenus burmannii entre otras. También son comunes hierbas como Ayenia dentata (Sterculiaceae), Gomphrena filaginoide (Amaranthaceae), Opuntia guatemalensis (Cactaceae) y Wissadula periplocifolia (Malvaceae). Como parte del bosque seco también se encuentra los zonzocuitales, que son sitios con suelos arcillosos propensos a anegarse en la época lluviosa (Apinet-INTA. (2000), Paguaga y Soto, (2010), Bazzurro, D. (1999), Carvallo. G,O. (2009), Fundación Nicaragüense para el Desarrollo Sostenible (2010), Pietronave, H. (2001).

\section{NotAS DE AUTOR}


La floración de árboles, arbustos y hierbas constituye elementalmente la fuente principal y única en todo caso de la alimentación de las abejas (Aphis melifera) en cualquier parte del planeta y hasta la actualidad no existen sustitutos apropiados para proveer la nutrición correcta de las abejas. Las abejas obtienen el néctar que es la fuente primaria de carbohidratos de su dieta y del polen de las flores obtienen importantes fibras y proteínas que formarán parte de sus estructuras físicas y morfológicas (Duttmann et al. (2013), Lúquez, F; y Valle, M. (2008), Mario R. Quiñónez[EF12] (2012).

La floración silvestre se da en diferentes meses del año, dependiendo del régimen y distribución de las lluvias en la zona de estudio. Según un estudio previo realizado por el Jardín Botánico de la UNAN-León reportado en la colección de árboles y arbustos de Occidente, las especies de plantas poliníferas y nectíferas que se encuentran en la zona de estudio "Pilas-El hoyo" pertenecen a las siguientes familias: Acantácea, Amarantácea, Anacardiácea, Asterácea, Bignoniácea, Bixácea, Boraginácea, Combretácea, Fabácea, lampacea, Malvácea, Mirtácea, Picramniácea, Plantaginácea, Poligonácea, Rubiácea, Sapindácea, Esterculiácea y Verbenácea (Paguaga y Soto,2010).

No se puede subestimar el aporte de numerosas y modestas plantas cultivadas y silvestres que no son aportadoras directas a las cosechas de miel, pero juegan un importante papel en el sostenimiento de las colonias en los períodos intercosechas. Tampoco los cítricos, eucaliptus, ajonjolí, girasol, etc., que pueden contribuir, siempre que se tenga en cuenta el peligro que representan las actividades fitosanitarias (fumigación con pesticidas) que se realizan en muchos cultivos con total ignorancia o negligencia respecto a la protección de estos valiosos polinizadores, (Pymerural. (2012), Apinet - INTA, (2000), Laboratorio de plantas vasculares, (2006).

A pesar de las condiciones ecológicas favorables para la floración de las especies melíferas, ocurre el fenómeno sistemático conocido como "la alternancia en la secreción del néctar". Pudiera definirse este término, como aquella manifestación por la cual las especies de plantas nectaríferas secretan, en determinados años, una cantidad de néctar mayor a la de otros años. En un mismo año, incluso, esta secreción puede llegar a ser nula para una o varias especies de plantas.

Las causas del comportamiento señalado deben buscarse, precisamente, en el complejo ecológico que actúa en la región y puede influir positiva o negativamente en la conducta nectaria de las plantas silvestres o cultivadas. Así, los apicultores, al hacer el resumen productivo del año, éste resulta bueno, regular o malo (Duttmann et al, 2013.)

León es una de las ciudades más antiguas de Nicaragua y es uno de los lugares donde más se ha desarrollado la actividad agrícola, lo que ha llevado a una disminución de las áreas boscosas y de sus árboles maderables. Vale la pena mencionar que en León se han recolectado varias especies de plantas reportadas como nuevos registros para la flora de Nicaragua que solo se conocen de un lugar específico de esta ciudad.

En León, los remanentes de bosque seco mejor conservados se encuentran en el Jardín Botánico Ambiental de la UNAN-León y el parque Arlen Siu. Además, en la ciudad hay sitios que podrían ser protegidos y conservados, como los remanentes de bosque en el margen sur del río Chiquito, donde está el Centro de Iniciativas Medioambientales de la Ciudad (CIMAC). (Paguaga y Soto, 2010).

Los apicultores carecen de conocimiento sobre las características de la floración de importancia apícola, siendo este fundamental para la conducción racional del apiario ya que constituye el recurso con que cuentan las abejas para alimentarse y producir (Tercero Martínez. X.L, Sequeira Castillo.C.Y. (2016), Centeno Keyling y Rodríguez Norma (2016), Rosillo H. 2010), Silva, L.M.; Restrepo, S. (2012), Rojas M, (2002) .

La flora es la que define la alternativa productiva (miel, cera, polen, jalea real, propóleos, etc.), el volumen de la producción y las características del producto. A su vez, el tipo de floración determinara la estrategia a usarse en los apiarios en cuanto al suplemento alimenticio y el manejo de las poblaciones de abejas. (ApinetINTA. (2000), Meyrat \& Alain. (2016)).

Mediante la presente investigación los apicultores tendrán un mayor conocimiento de las características de las especies melíferas y su disponibilidad durante el año. 


\section{Materiales y MÉTOdos}

\subsection{Ubicación y área de estudio.}

El estudio se llevó a cabo en el área protegida Complejo Volcánico Cerro Negro - Pilas - El Hoyo, ubicada aproximadamente a $30 \mathrm{~km}$ al este de la ciudad de León y está conformado por varias formaciones volcánicas que comparten la misma base, siendo el más destacado el Volcán Pilas, con su cráter activo el Hoyo (1089 msnm). Hacia el oeste se levanta el Cerro Negro $(726 \mathrm{msnm})$, siendo este el volcán más joven activo del país. Hacia el sur se alza el cono truncado y apagado del volcán Asososca o Ajusco ( $818 \mathrm{msnm}$ ), en cuya base se abre la laguna cratérica, también llamada Laguna del Tigre, desde cuyo borde se observa uno de los más bellos paisajes del conjunto volcánico formado por el Momotombo, Momotombito, Pilas - El Hoyo, la propia laguna y el lago de Managua con la Península de Chiltepe.

La temperatura anual en la base de estos volcanes es de unos $28^{\circ}$ Centígrados y disminuye aproximadamente en un grado por cada 150 metros de elevación. Alrededor del cráter principal de El Hoyo hay formaciones azonales afectadas por la pendiente arenosa y la presencia permanente de una fumarola. La propia cumbre se encuentra afectada por el calor interno del volcán que limita el desarrollo de la vegetación sobre las laderas externas[EF2].

La mayor cantidad de los apiarios estacionarios ubicados en el sector del Complejo Volcánico Cerro Negro - Pilas - El Hoyo, pertenecen a las siguientes cooperativas:

Cooperativa multisectorial de los Terreros (COOPAMIT), Cooperativa de Apicultores Conservadores del Medio Ambiente Manos Unidos R.L. (CACOMAMUN), Cooperativa de Apicultores de los Maribios de Miramar Villa Unión, R. L. (COOPAMIRV), Mujeres de Xóchitl Acalt y apicultores de la Unión de Cooperativas Agropecuarias de El Sauce (UCASA).

\subsection{Tipo de estudio.}

Descriptivo de corte longitudinal ya que se evaluó las variables a lo largo del tiempo.

\subsubsection{Fases del estudio.}

El presente estudio se dividió en dos fases:

Primera fase:

Consistió en realizar un Diagnóstico Rural Participativo (DRP) con los integrantes de la Cooperativa multisectorial de los Terreros (COOPAMIT), Cooperativa de Apicultores Conservadores del Medio Ambiente Manos Unidos R.L. (CACOMAMUN), Cooperativa de Apicultores de los Maribios de Miramar Villa Unión, R. L. (COOPAMIRV), Mujeres de Xóchitl Acalt, utilizando herramientas participativas; cuyo objetivo fue obtener información de los conocimientos que manejan sobre las especies de aprovechamiento apícola del lugar, cuáles son las especies más utilizadas por las abejas, el beneficio de las mismas y el grado de importancia para los apicultores.

Segunda fase:

Consistió en realizar los muestreos para determinar la flora de aprovechamiento apícola, determinar el uso de la tierra presente en los alrededores de los apiarios y verificar los meses de floración de las especies repitiendo esta actividad durante los meses que duró el estudio; en dichos muestreos se utilizaron las metodologías diseñadas especialmente para la realización de este estudio.

3.3. Análisis de los datos. 
Los datos recolectados en los formatos de muestreo, fueron transferidos a una base datos en Excel. Los análisis estadísticos consistieron en: graficas de barras y pastel.

\section{Resultados y Discusión.}

\subsection{Resultados de la identificación de las especies florísticas de la zona del complejo volcánico Pilas el Hoyo.}

En el resultado de la identificación de las especies florísticas de la zona del complejo volcánico Pilas el Hoyo (tabla1, tabla2, tabla3 ver anexos) se identificaron 89 especies, pertenecientes a 39 familias, según su estrato vegetal estas especies se dividen en: 59 especies de árboles siendo este el $66.29 \%, 15$ especies de arbustos con el $16.85 \%$ y 15 especies de hierbas con el $16.85 \%$.

En el censo florístico realizado en la comunidad del Tule Municipio San Miguelito, Rio San Juan. Octubre - 2016., se demostró que en la zona el tipo de vegetación presente está conformada en un $65.6 \%$ por árboles de diferentes portes, los arbustos representan el $20 \%$ y el restante $14.4 \%$ está conformada por especie de tipo rastrera, semiarbustivas, diversas hierbas del tipo rastreros y semiarbustivas, además de palmeras.

La zona del complejo volcánico Pilas el Hoyo presenta una diversidad de especies que ponen a disposición el alimento (néctar y polen) para las abejas. Este mayor porcentaje de árboles presente en ambos estudios se debe a que en las dos zonas de estudio se muestra un bosque seco tropical el cual está constituido según Hernández-Salas \& Quesada-Monge, (2000), por dos estratos de árboles. Un primer estrato denominado dosel con árboles de 20 a 30 metros de alto, el segundo estrato está formado por el sotobosque con árboles de 10 a 20 metros de alto y el nivel de los arbustos tiene de 2 a 5 metros de altura.

\subsection{Resultados distribución y dominancia la flora para aprovechamiento apícola de la zona del complejo volcánico pilas el hoyo .}

En el resultado de las familias de árboles con alta dominancia (grafico1), la familia con mayor número de individuos muestreados dentro de todo el complejo volcánico Pilas- el Hoyo es la Bignoniácea con 897 individuos muestreados, seguido por las familias Myrtaceae y Fabácea con más de 738 y 659 individuos muestreados respectivamente, por ultimo están las familias Ebenácea y Boraginácea con más de 100 individuos muestreados cada una.

Según Paguaga y Soto (2010) las especies de plantas poliníferas y nectíferas que se encuentran en la zona de estudio "Pilas-El hoyo" pertenecen a las siguientes familias: Acantácea, Amarantácea, Anacardiácea, Asterácea, Bignoniácea, Bixácea, Boraginácea, Combretácea, Fabácea, lampacea, Malvácea, Mirtácea, Picramniácea, Plantaginácea, Poligonácea, Rubiácea, Sapindácea, Esterculiácea y Verbenácea.

Como podemos ver hay una similitud entre las familias presentes en este estudio (gráficas 1,2 y 3 ) y el realizado por Paguaga y Soto, 2010. Lo que se debe a que estas familias son comunes del bosque seco tropical. 


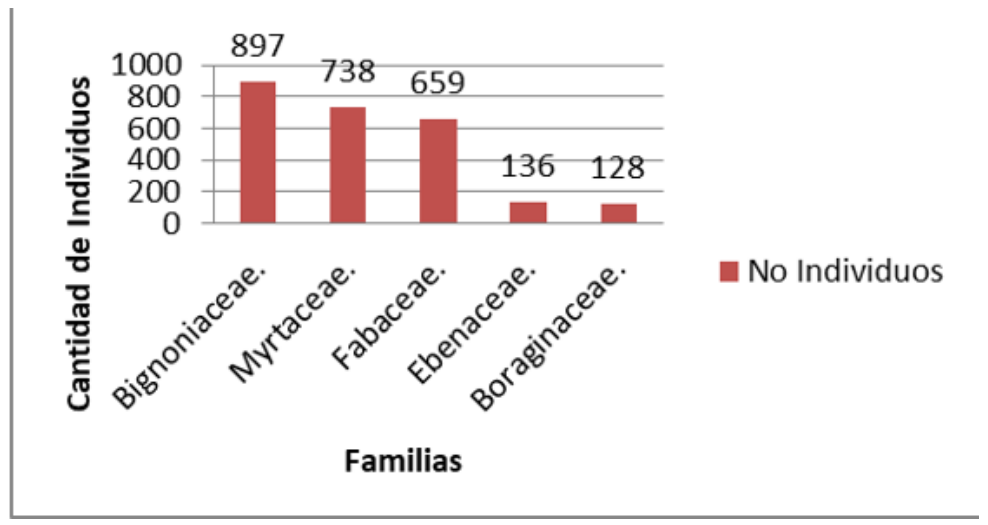

GRAFICO 1

Familias arbóreas con alta dominancia

\subsection{Calendario floral de las especies de la flora de aprovechamiento apícola de la zona del complejo volcánico Pilas el Hoyo.}

En el complejo volcánico Pilas- El Hoyo se encontró una mayor floración entre los meses de Diciembre a Mayo, de Junio a Noviembre hay una escasez de floración existen 14 especies que florecen durante todo el año y algunos cultivos presentes en la zona lo que hace notar la importancia de estas y los cultivos para la alimentación de las abejas durante el periodo de escasez floral.

En el resultado de las familias de árboles con abundancia media (Grafico2), la familia con mayor número de individuos muestreados dentro de todo el complejo volcánico Pilas- el Hoyo es la Cesalpinaceae con de 95 individuos, seguida por las familias Lamiaceae, Meliaceae con 75 y 38 individuos muestreados respectivamente, Burseráceae, Mimosacea y Anacardiacea con más de 20 individuos cada una, Bixaceae con 20 individuos, Sapindaceae, Sterculiaceae y Polygonaceae con más de 10 individuos y Apocynacea con 10 individuos cada una.

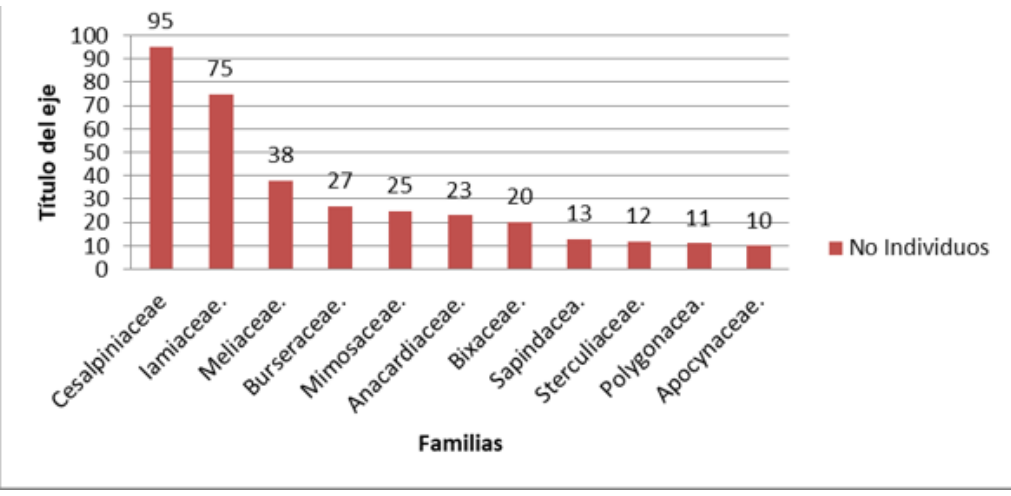

\section{GRAFICA 2}

Familias de árboles con abundancia media

En el resultado de las familias de árboles con baja abundancia (Grafico3), la familia con mayor número de individuos muestreados dentro de todo el complejo volcánico Pilas- el Hoyo es la Tiliáceae con 7 individuos, seguida por la Simaroubaceae y Rubiaceae con 5 y 3 individuos muestreado respectivamente, las familias Sapotaceae, Moraceae, Cecropiacea, Malpighiacea y Annonaceae con 1 individuo muestreado cada una. 


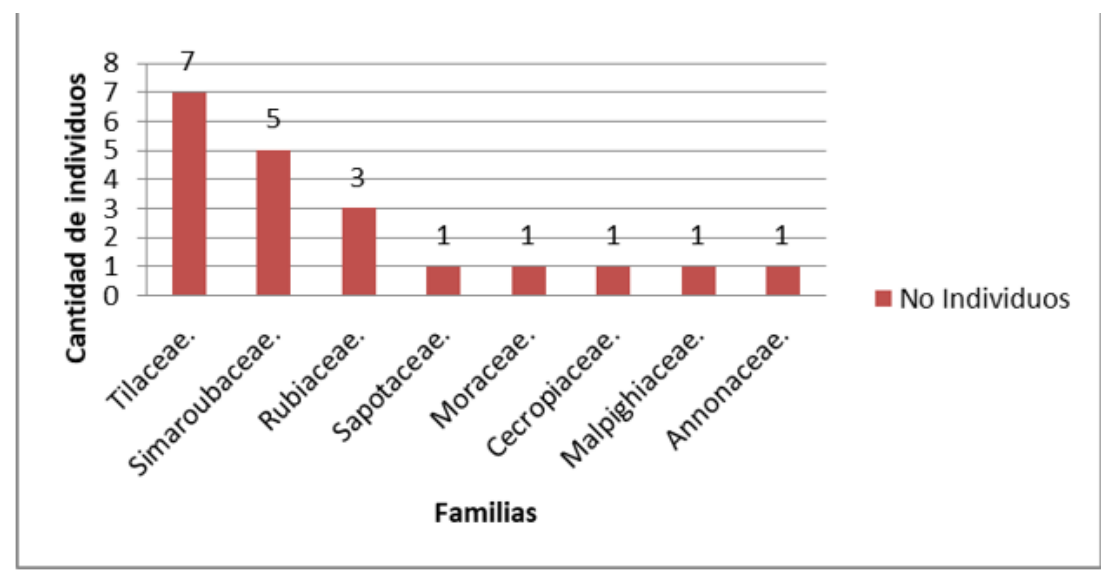

GRAFICO 3.

Familias de árboles con baja dominancia

En el resultado de las especies de árboles con mayor dominancia (Grafico4), la familia con mayor número de individuos muestreados dentro de todo el complejo volcánico Pilas- el Hoyo es el Sardinillo (Tecoma Stan) con 830 individuos muestreados seguido por el Eucalipto (Eucalyptus camaldulensis) y Chaperno (Lonchocarpus rugosus) con 685 y 416 individuos muestreados respectivamente, Chocoyito (Diospyros salicifolia) con 136 individuos, Madero negro (Gliricidia sepium) con 110 individuos, Laurel (Cordia alliodora) con 76 individuos, Brasil (Haematoxylum brasiletto) con 66 individuos, Tigüilote (Cordia dentata) con 51 individuos, Michiguiste (Pithecellobium dulce) con 49 individuos, Cortez (Tabebuia ochracea) con 44 individuos, Jícaro (Crescentia cujete) con 22 individuos, Quebracho (Lysiloma auritum) con 16 individuos, Genizaro (Samanea/Albizia saman) con 2 individuos, Muñeco (Cordia collococca) y Roble (Tabebuia rosea) con 1 individuo cada uno.

A pesar que las especies de Sardinillo (Tecoma Stan) y el Eucalipto (Eucalyptus camaldulensis) no son originarias de este país, estas fueron introducidas en la zona del complejo volcánico "Pilas - el Hoyo "como parte del Proyecto de Los Maribios de la FAO, en respuesta paliativa para tratar de recuperar los bosques naturales. (Marena-protierra-cba). La dominancia mostrada del Eucalipto (Eucalyptus Camaldulensis) es debido a que en la zona del muestreo se encontraba una plantación de este, cabe destacar que si esta plantación no es bien manejada y es acabada por los pobladores, luego se empezará a deforestar las demás especies presentes en el complejo volcánico "Pilas - el Hoyo" lo que hace de suma importancia cuidar estas plantaciones. En cuanto al Sardinillo (Tecoma Stan) su dominancia es debido a que esta especie del tipo invasora las cuales según Carvallo (2009), presentan una alta densidad y gran número de individuos, se establecen en grandes áreas en unas pocas generaciones, tienen una reproducción y crecimiento rápido, y pueden llegar a producir la extinción de especies nativas. 


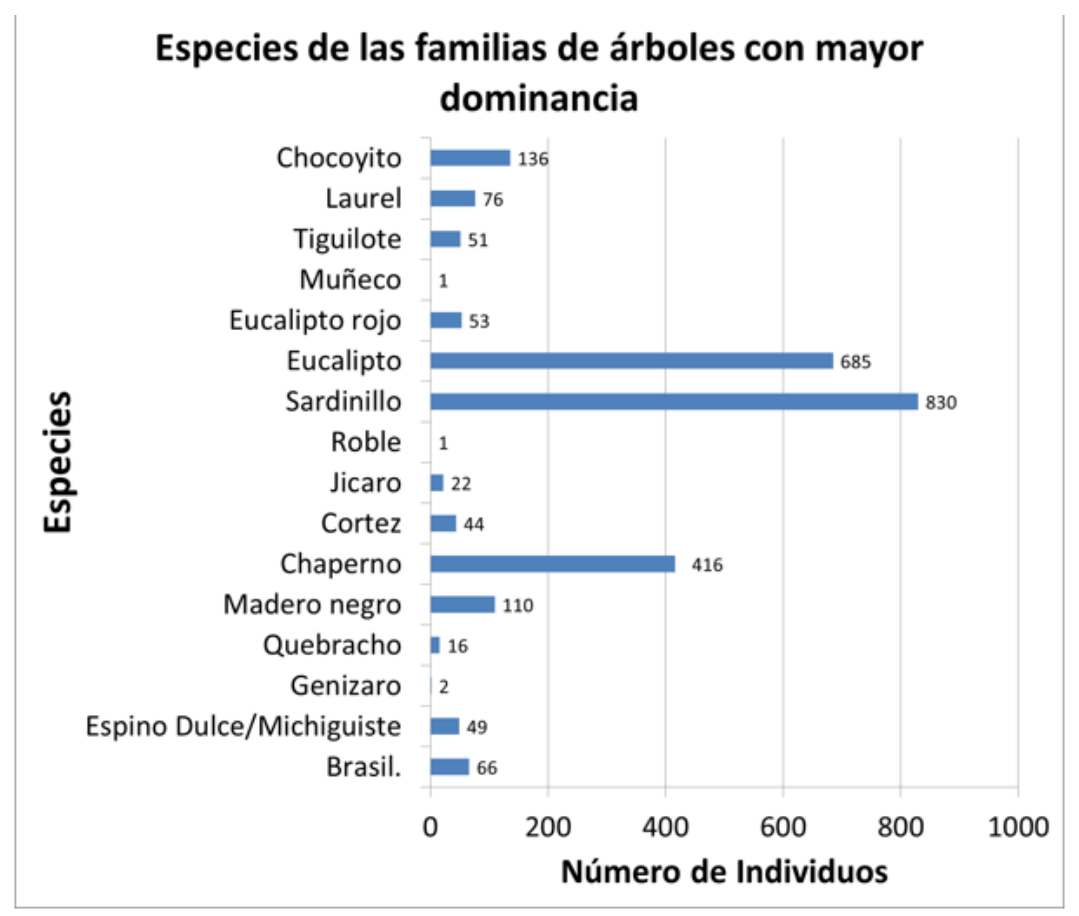

GRAFICO 4.

Especies de árboles con mayor dominancia

En el resultado de las especies de árboles dominantes por apiario (Grafico5), la especies con mayor número de individuos muestreados dentro del apiario la teca son el Sardinillo (Tecoma stan) con 600 y Eucalipto (Eucalyptus Camaldulensis) con 564 individuos muestreado respectivamente, en el apiario la Diabla la especie con mayor cantidad de individuos muestreados es el Chaperno (Lonchocarpus rugosus) con 261 individuos seguido por el Madero negro (Gliricidia sepium) con 97 individuos, en el apiario termopila 2 la especie más dominante es el Chaperno (Lonchocarpus rugosus) con 136 individuos muestreados seguido del Chocoyito(Diospyros salicifolia) con 57 individuos y Laurel (Cordia alliodora) con 14 individuos, En el apiario la piedra la especie más dominante fue el Sardinillo (Tecoma stan) con 94 individuos muestreados seguido del Laurel (Cordia alliodora) con 27 individuos, en el apiario la laguna la especie más dominante fue el Eucalipto (Eucalyptus Camaldulensis) con 75 individuos seguida por el Sardinillo (Tecoma stan) con 66 individuos, en el apiario termopila 1 la especie más dominante fue el Cortez (Tabebuia ochracea) con 29 individuos seguido por el Chaperno (Lonchocarpus rugosus) con 7 individuos. 


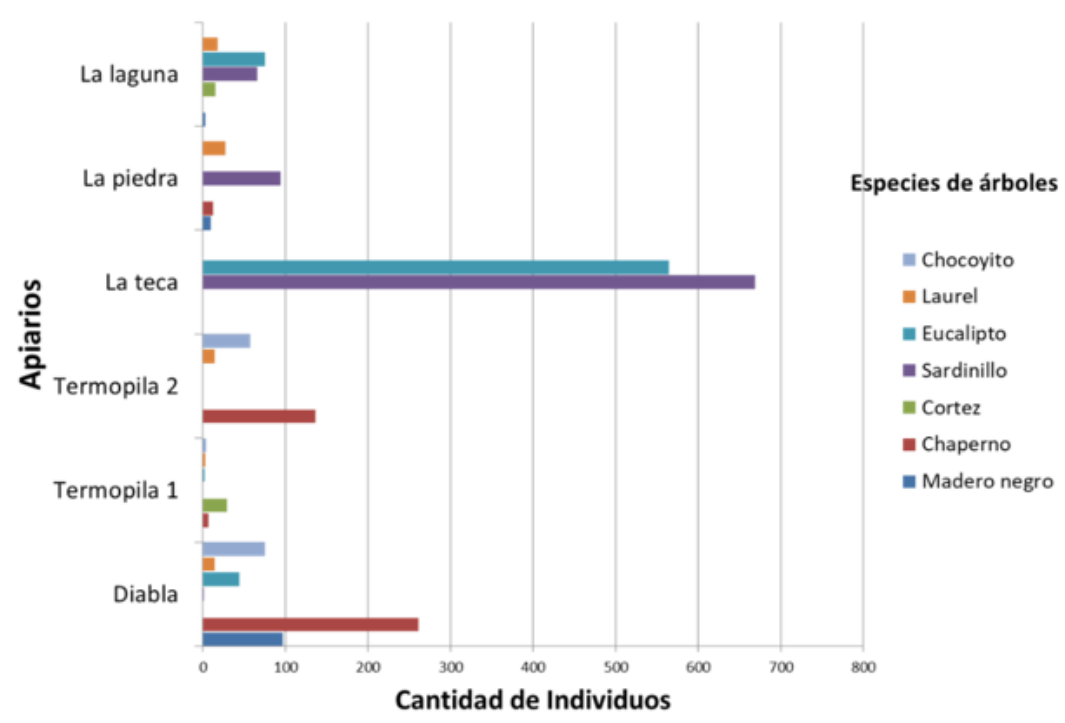

GRAFICO 5.

Especies de árboles dominantes por apiario

En el resultado de familias de arbustos más dominantes (Grafico 6), la familia con mayor cantidad de individuos muestreados fue la Verbenácea con 1658, seguida por la Boraginaceae con 703 individuos muestreados, Fabaceae con 383 individuos, Apocynaceae con 226 individuos y Solanaceae con 168 individuos.

Como podemos ver hay una similitud entre las familias presentes en este estudio (gráficas 6 y 7 ) y el realizado por Paguaga y Soto, 2010. Lo que se debe a que estas familias son comunes del bosque seco tropical.

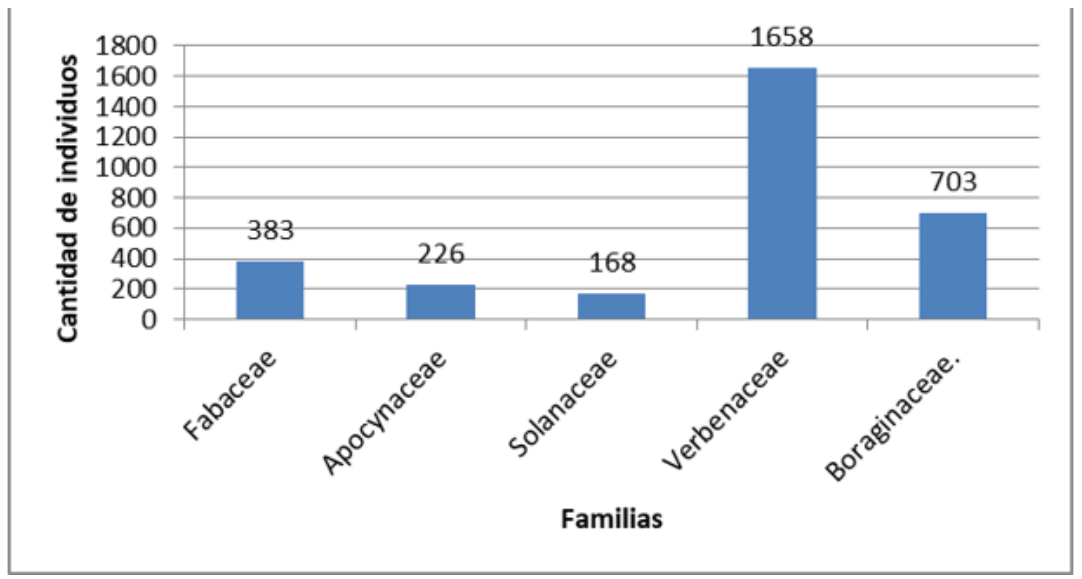

GRAFICO 6.

Familias de arbustos más dominantes

En el resultado de las familias de arbustos menos dominantes (Grafico 7), la familia con mayor cantidad de individuos muestreados es la Flacourtacea con 37, seguida por la Theoprhastaceae con 19 individuos, Combretacea[EF5] con 13 individuos, Malvaceae con 8 individuos y Capparaceae con 2 individuos. 


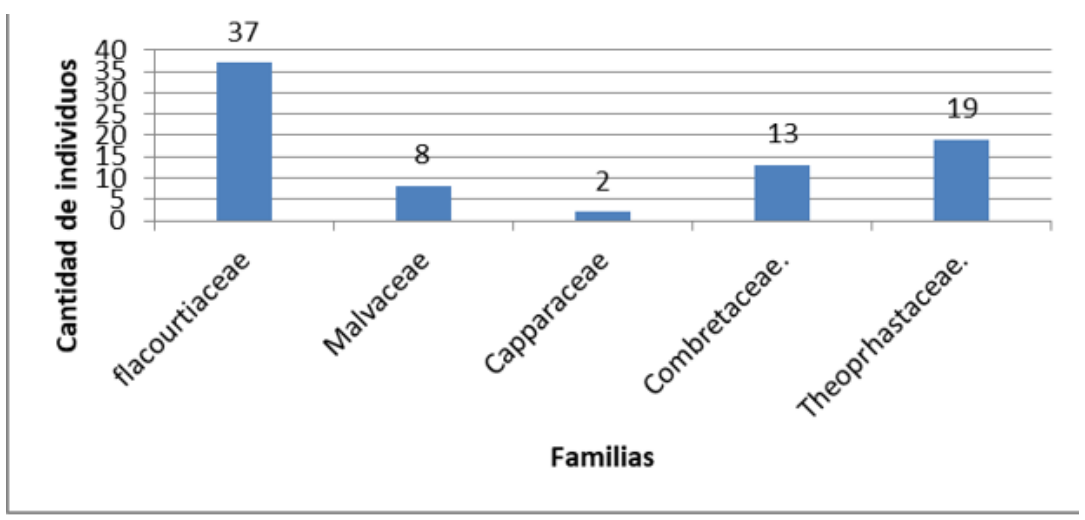

\section{GRAFICO 7.}

Familias de arbustos menos dominantes

En el resultado de especies arbustivas más abundante (Grafico 8), la especie con mayor abundancia fue el botoncillo o cinco negritos (Lantana urticifolia) con 1658 individuos muestreados, seguida por el Achopaste (Varronia inermis) con más de 703 individuos, Cornizuelo (Vachellia collinsii) con 232 individuos, Cachito (Stemmadenia obovata) con 221 individuos, lavaplato (Solanum erianthum) con 159 y Ron ron con 151 individuos cada una.

La dominancia del botoncillo o cinco negritos (Lantana urticifolia) puede deberse a la facilidad de propagación y adaptación que presenta este especie en climas como en el presente en la zona del complejo volcánico Pilas - el hoyo, es una especie atractiva para las abejas por sus colores y es una fuente de néctar.

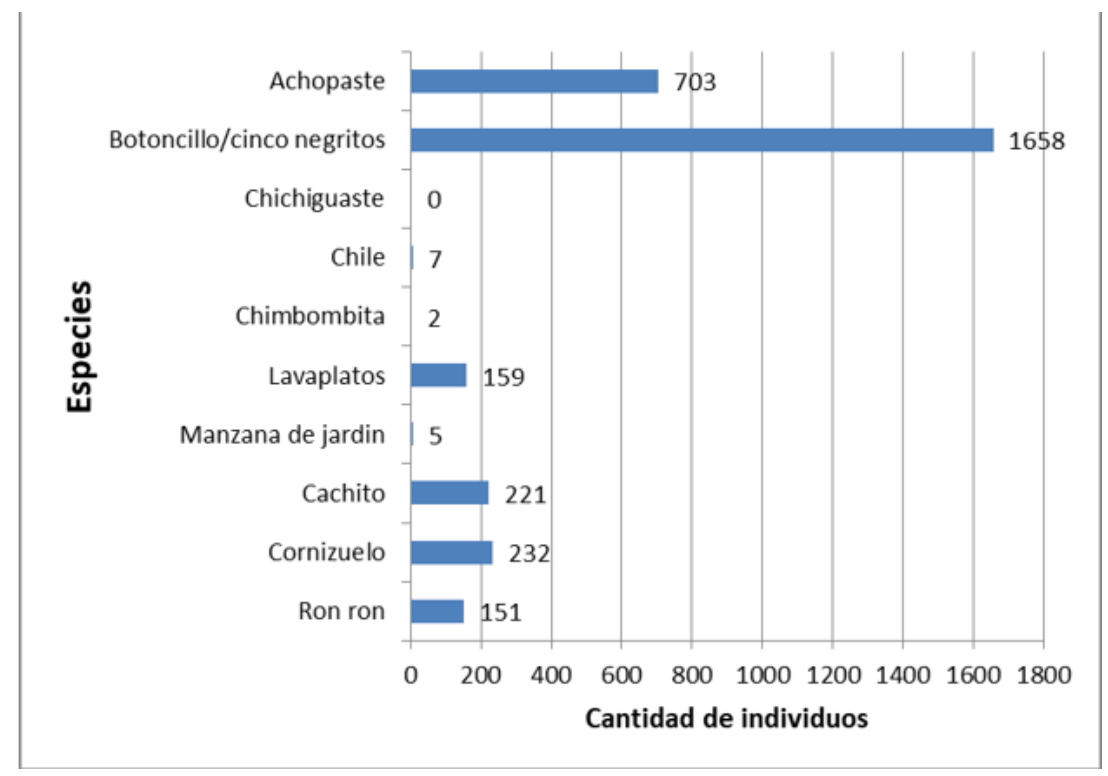

GRAFICO 8.

Especies arbustivas más abundantes

En el resultado de las especies de arbustos más dominan por apiario (Grafico 9), en el apiario la Teca la especie más dominante es el Botoncillo (Lantana urticifolia) con 1043 individuos muestreados seguido por el Cornizuelo (Vachellia collinsii) con 26 individuos y Cachito (Stemmadenia obovata) con 11 individuos, en el apiario Termopila 1 la especie más dominante es el Achopaste (Varronia inermis) con 678 individuos muestreados seguido por el Cachito (Stemmadenia obovata) y el Cornizuelo (Vachellia collinsii) con 65 individuos cada uno, en el apiario la piedra la especie más dominante es el Botoncillo (Lantana urticifolia) con 534 individuos, en el apiario la Yuca la especie más dominante es el Cornizuelo (Vachellia collinsii) con 141 
individuos muestreados seguido por el Ron ron (Senna skinneri) con 129 individuos y el lavaplatos (Solanum erianthum) con 125 individuos, En el apiario la diabla la especie más dominante es el Cachito (Stemmadenia obovata) con 85 individuos, seguido por el Botoncillo (Lantana urticifolia) con 37 individuos y el Achopaste (Varronia inermis) con 25 individuos, En el apiario la Laguna la especie más dominante es el Cachito (Stemmadenia obovata) con 41 individuos, seguido por el Ron ron (Senna skinneri) con 21 individuos y Botoncillo (Lantana urticifolia) con 16 individuos, En el apirio Termopila 2 la especie más dominante es el Botoncillo (Lantana urticifolia)con 19 individuos, seguida por el lavaplatos (Solanum erianthum) con 12 individuos.

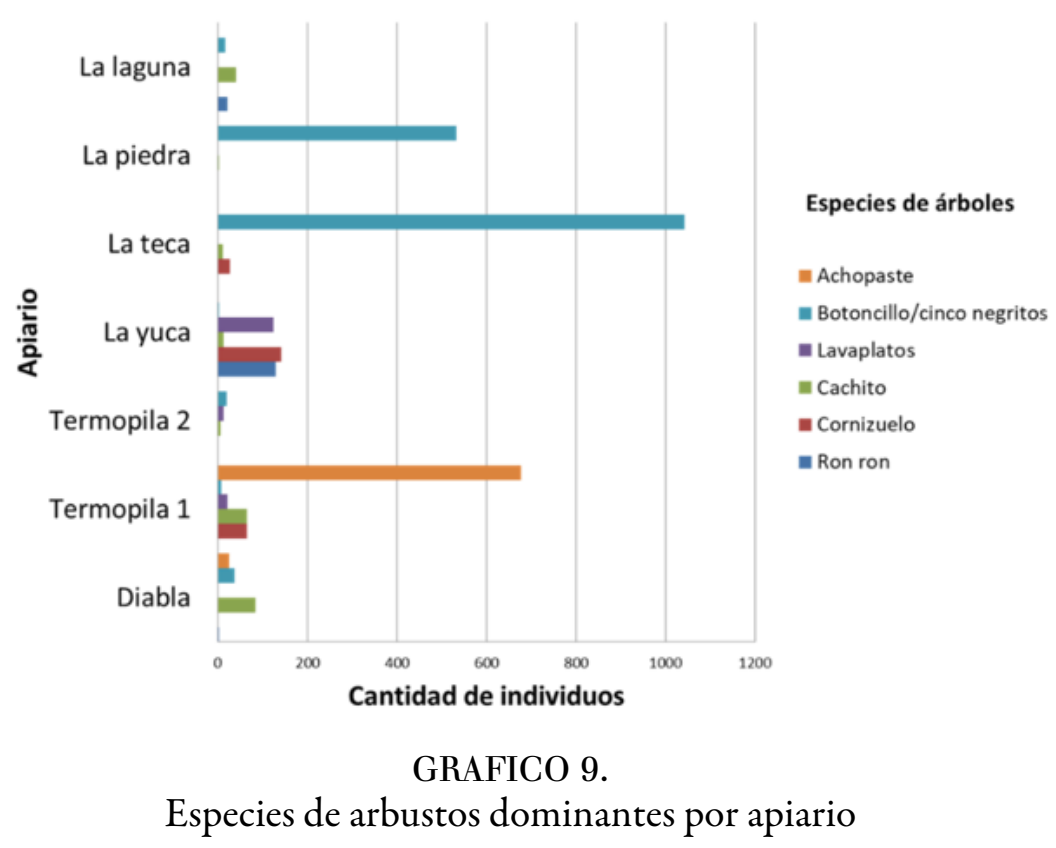

En el resultado de las familias de hierbas mas dominantes (Grafico 10), la familia mas dominante es la Plantaginacea con 17,500 individuos muestreados seguido por la Malvacea con 15,454 individuos, la familia Sterculiaceae con 11,000 individuos, la familia Lamiaceae con 4,076 y la familia Convolvulacea con mas de 1,238 individuos.

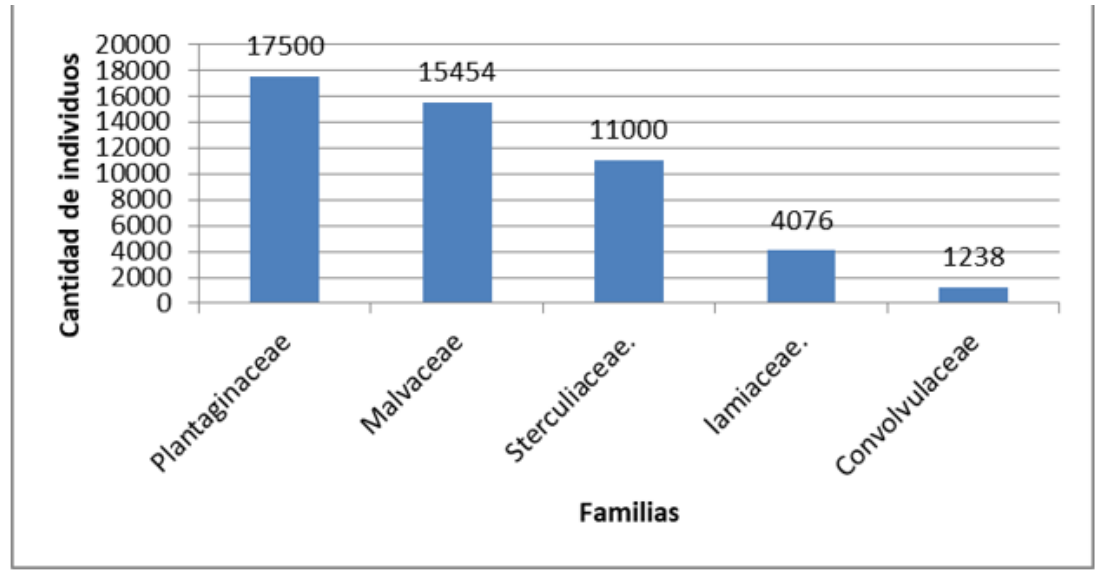

GRAFICO 10.

Familias de hierbas más dominantes 
En el resultado de las familias de hierbas menos dominantes (Grafica 11), la familia con mayor número de individuos muestreados es la Acanthaceae con 202, seguido por la Verbenaceae con 25 individuos, Fabacea con 3 individuos y Amarantaceae con 2 individuos.

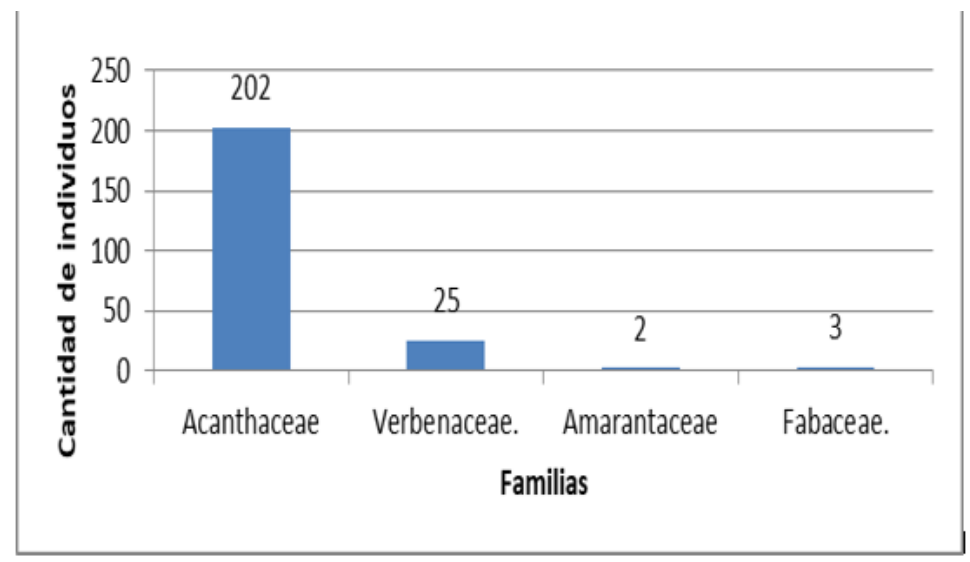

GRAFICO 11.

Familias de hierbas menos dominantes

En el resultado de las familias de hierbas de mayor abundancia (Grafico 12), la especie con mayor abundancia es la Escoba amarilla (Scoparia dulcis I) con 17,500 individuos muestreados seguida por la escoba lisa (Waltheria indica l) con 11,000 individuos, la Escoba pachona (Waltheria indica) con 9000 individuos, Escoba blanca (Sida acuta) con 6454 individuos, el chan (Hyptissuaveolens) con 4,050 individuos, la campanita (Ipomoea sp.) con 1,238 individuos y el Orégano (Origanum sp) con 26 individuos.

Estas especies son de importancia ya que suplen de alimento a las abejas y su número es bastante alto, el mayor número de estas fue encontrado en las áreas de barbecho (áreas agrícolas en reposo).

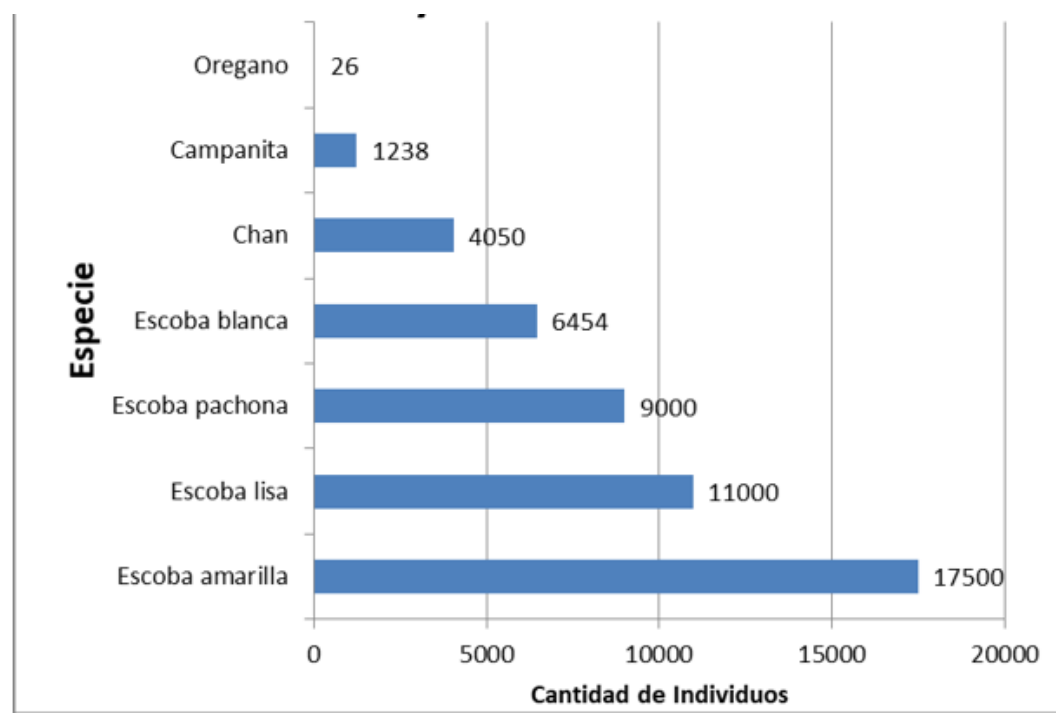

GRAFICO 12

Especies de hierbas de mayor abundancia

En el resultado de las especies de hierbas dominantes por apiario (Grafico 13), en el apiario Termopila 1 la especie más dominante es la escoba lisa (Waltheria indica l) [EF6] con 9000 individuos muestreados seguida por Escoba amarilla (Scoparia dulcis I) con 8000 individuos, en el apiario la piedra la especie más dominante es la Escoba pachona (Waltheria indica) con 9000 individuos seguida por escoba lisa (Waltheria indical) con 2000 individuos y la campanita (Ipomoea sp.) con 1200 individuos, en el apiario la yuca la especie más 
dominante es la escoba amarilla (Scoparia dulcis I) con 6000 individuos, en el apiario Termopila 2 la especie más dominante es Escoba blanca (Sida acuta) con 4000 individuos seguida por el chan (Hyptis suaveolens) con 2000 individuos, en el apiario la teca hay una homogeneidad entre Escoba amarilla (Scoparia dulcis I), chan (Hyptis suaveolens) y Escoba blanca (Sida acuta) con 2000 individuos cada una, en el apiario la laguna la especie más dominante es por Escoba amarilla (Scoparia dulcis I) con 1500 individuos, seguida por Escoba amarilla (Scoparia dulcis I) con 450 individuos y la campanita (Ipomoea sp.) con 34 individuos.

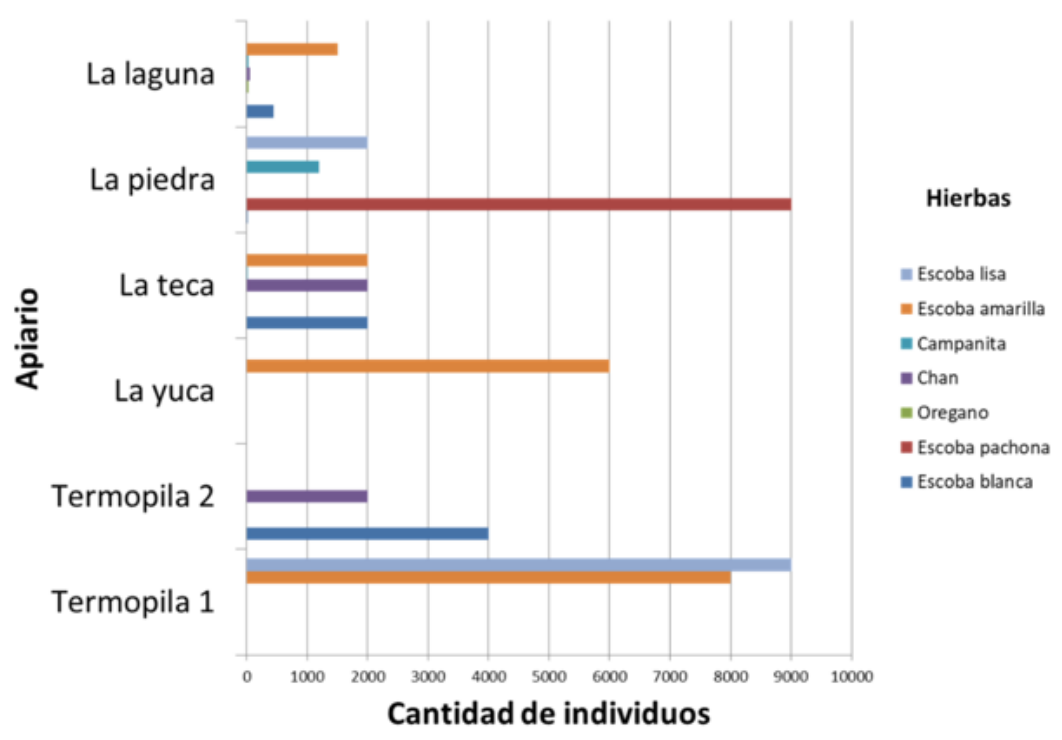

GRAFICO 13.

Especies de hierbas dominantes por apiario

\subsection{Resultados Caracterización del uso actual de la tierra de la zona del complejo volcánico Pilas el Hoyo.}

En el resultado de la distribución del uso de la tierra en el apiario la piedra (Grafico 14) se encontró un mayor porcentaje de área silvestre con 34\% seguido por 21\% de barbecho, 13\% de maní, 9\% de área habitada, 7\% de potrero, $7 \%$ de frijol blanco, $5 \%$ de otros (Pasto, yuca, melón, sorgo, ayote, frutales y ajonjolí) y un $4 \%$ de Maíz.

El apiario la piedra cuenta solo con un 34\% de área silvestre ya que está ubicado cerca del asentamiento de Miramar, pero este bajo porcentaje se ve compensado con la diversidad de cultivos que a su vez ofrecen néctar (Melón y frutales) y polen (Maíz, sorgo, ajonjolí, etc) como fuente de alimento a las abejas. 


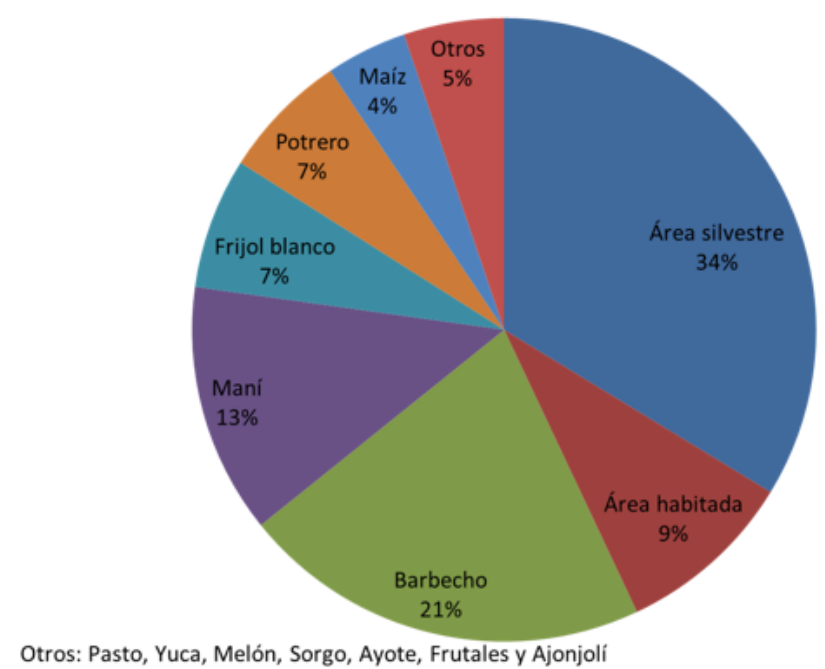

GRAFICO 14.

Distribución del uso de la tierra en el apiario la piedra

En el resultado de la distribución del uso de la tierra en el apiario las Diablas (Grafico 15), se encontró un mayor porcentaje de área silvestre con $75 \%$ seguido por el barbecho con $12 \%, 8 \%$ de maní, 3\% de otros (Pasto, yuca, melón, sorgo, ayote, frutales y ajonjolí) y un $2 \%$ de frijol blanco.

El apiario las Diablas cuenta con un mayor porcentaje de área silvestre lo que garantiza una variada oferta floral y por lo tanto alimenticia para las abejas.

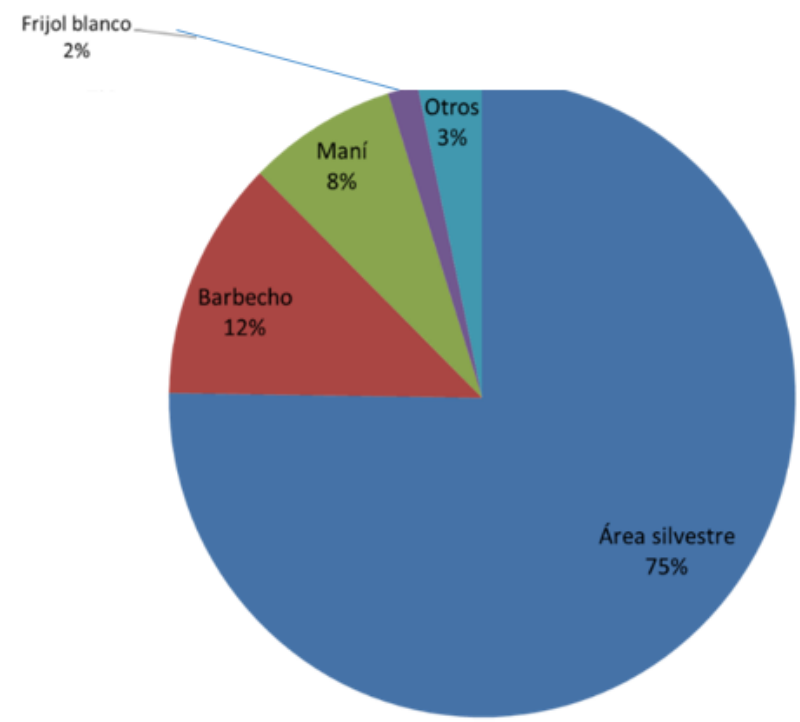

GRAFICO 15.

Distribución del uso de la tierra en el apiario las Diablas

En el resultado de la distribución del uso de la tierra en el apiario "La Laguna" (grafico 16) se encontró un mayor porcentaje de bosque silvestre con $63 \%$ seguido por el maní con $17 \%, 10 \%$ de pasto, $8 \%$ de barbecho y el Maíz con un 2\% de toda el area muestreada.

El apiario la laguna cuenta con una mayor área silvestre, que representa una variedad de floración de distintos tipos de plantas, cabe mencionar que el cultivo de maní tiene un área considerable en la zona ya que es el segundo con mayor porcentaje de uso, pese a que este cultivo es pecoreado por las abejas, el manejo convencional con pesticidas y fertilizantes químicos son nocivos para estas. 


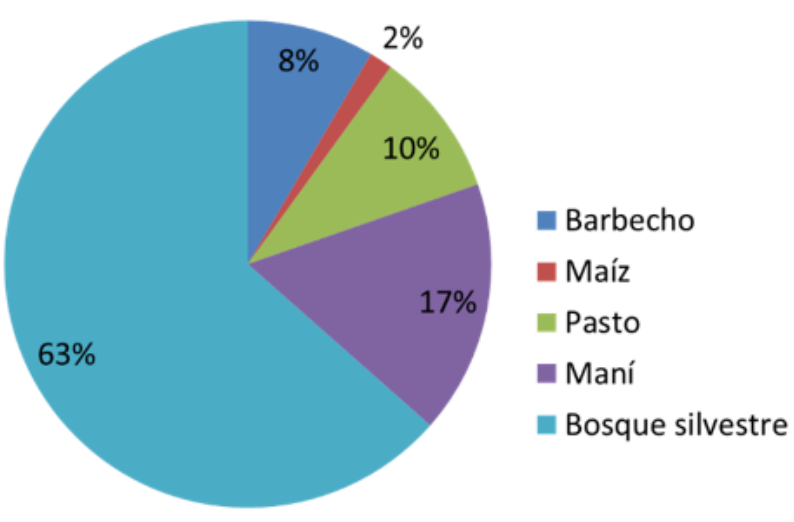

GRAFICO 16.

Distribución del uso de la tierra en el apiario la Laguna

En el resultado de la distribución del uso de la tierra en el apiario "La Teca" (Grafico 17) se encontró un mayor porcentaje de Eucalipto con 25\% seguido por el bosque silvestre con 23\%, 10\% de barbecho, Yuca y Maíz con $9 \%$ cada uno, Ajonjolí con 5\%, Potrero y pasto con $4 \%$ cada uno y Maní con 3\% de toda el área muestreada.

El mayor porcentaje de Eucalipto (Eucalyptus Camaldulensis) se debe a que hay presente una plantación de este cerca del apiario muestreado, esta especie es muy importante para la apicultura ya que ofrece néctar, polen y propóleos.

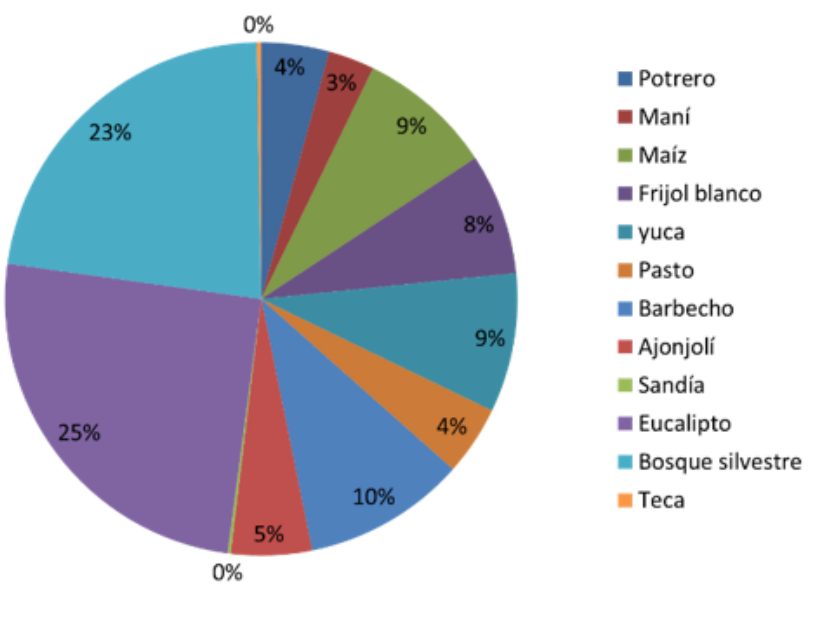

GRAFICO 17.

Distribución del uso de la tierra en el apiario la Teca

En el resultado de la distribución del uso de la tierra en el apiario "La Yuca" (Grafico 18) se encontró un mayor porcentaje de bosque silvestre y barbecho con $28 \%$ cada uno seguido por los cultivos de yuca con $16 \%$, Maní con 11\%, Frijol blanco y pasto con $7 \%$ cada uno y Ajonjolí con 3\% de toda el area muestreada. 


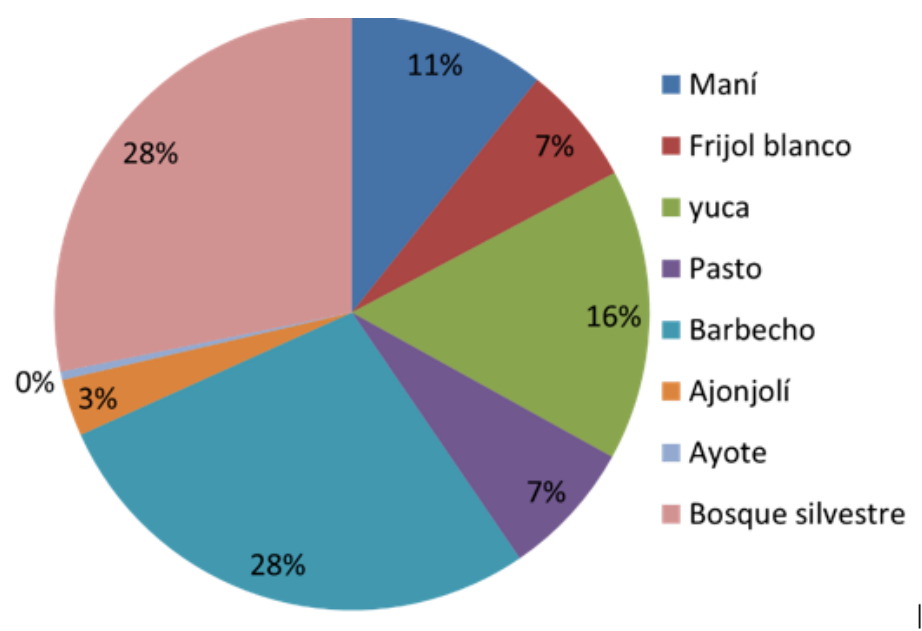

GRAFICO 18.

Distribución de uso de la tierra en el apiario la Yuca

En el resultado de la distribución del uso de la tierra en el apiario "Los Terreros 1" (Grafico 19) se encontró un mayor porcentaje de bosque silvestre constando con un $70 \%$ seguido de los cultivos de Maíz con 15\%, Caña con $9 \%$ y Sorgo con $6 \%$ de toda el área muestreada.

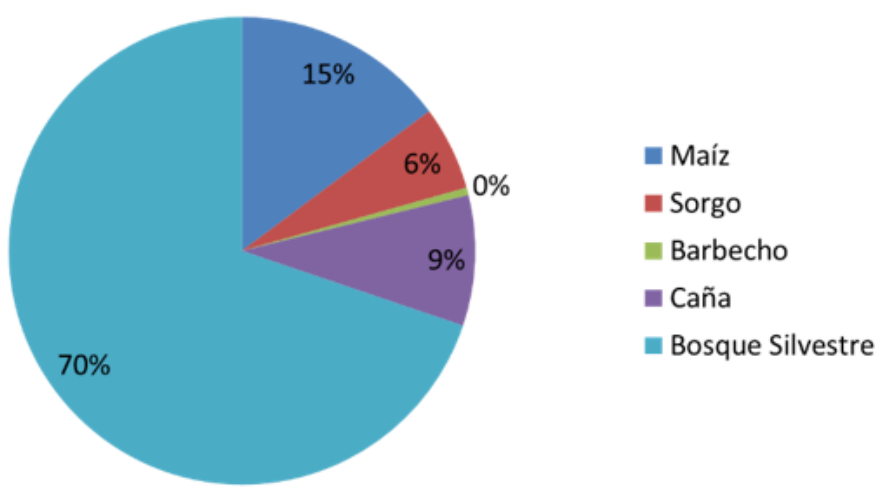

GRAFICO 19.

Distribución del uso de la tierra en el apiario los Terreros 1

En el resultado de la distribución del uso de la tierra en todo el complejo volcánico "Pilas - el Hoyo" (Grafico 21) se muestra un mayor porcentaje de bosque silvestre con el $53 \%$ seguido por el barbecho con $12 \%$, Maní $8 \%$, Maíz y Sorgo con 6\% cada uno, Eucalipto, frijol blanco y Yuca con 3\% cada uno, potrero y caña con $2 \%$, pasto y Ajonjolí con $1 \%$.

En el resultado de la distribución del uso de la tierra en el apiario "Los Terreros 2" (Grafico 20) se encontró un mayor porcentaje de bosque silvestre constando de $51 \%$ seguido por el cultivo de Sorgo con $26 \%$, el barbecho y el cultivo de Maíz con $9 \%$ cada uno, potrero con $4 \%$ y el jícaro con $1 \%$ de toda el área muestreada. 


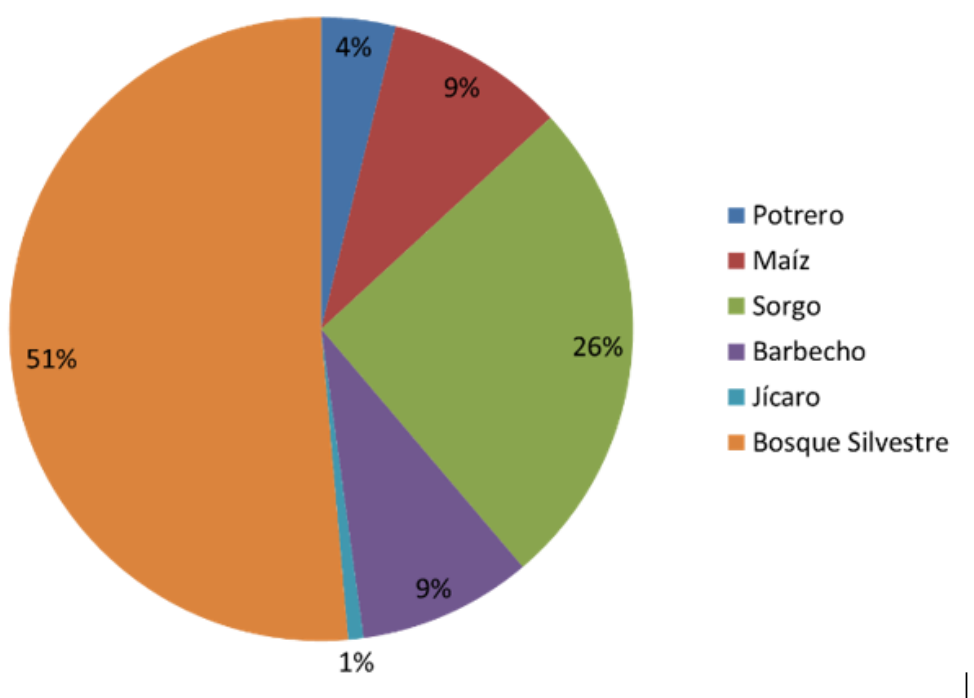

GRAFICO 20.

Distribución del uso de la tierra en el apiario los Terreros 2

Los mayores porcentajes que son el bosque silvestre y el barbecho, son los que ofrecen la mayor parte de la floración disponible entre árboles, arbustos y hierbas estas últimas con mayor presencia en las áreas de barbecho, el maní es el tercer porcentaje más alto del uso de la tierra lo que se puede tomar como un peligro para los apicultores, ya que este cultivo tiende a aumentar su área cultivable lo que conlleva expansión de la frontera agrícola y por ende la desforestación, además de presentar un manejo convencional implementando el uso de insecticidas y fertilizantes químicos nocivos para la vida de las abejas, el cultivo de Maíz es una buena fuente de polen que puede alimentar a las abejas durante el tiempo con menor floración (Grafico 21).

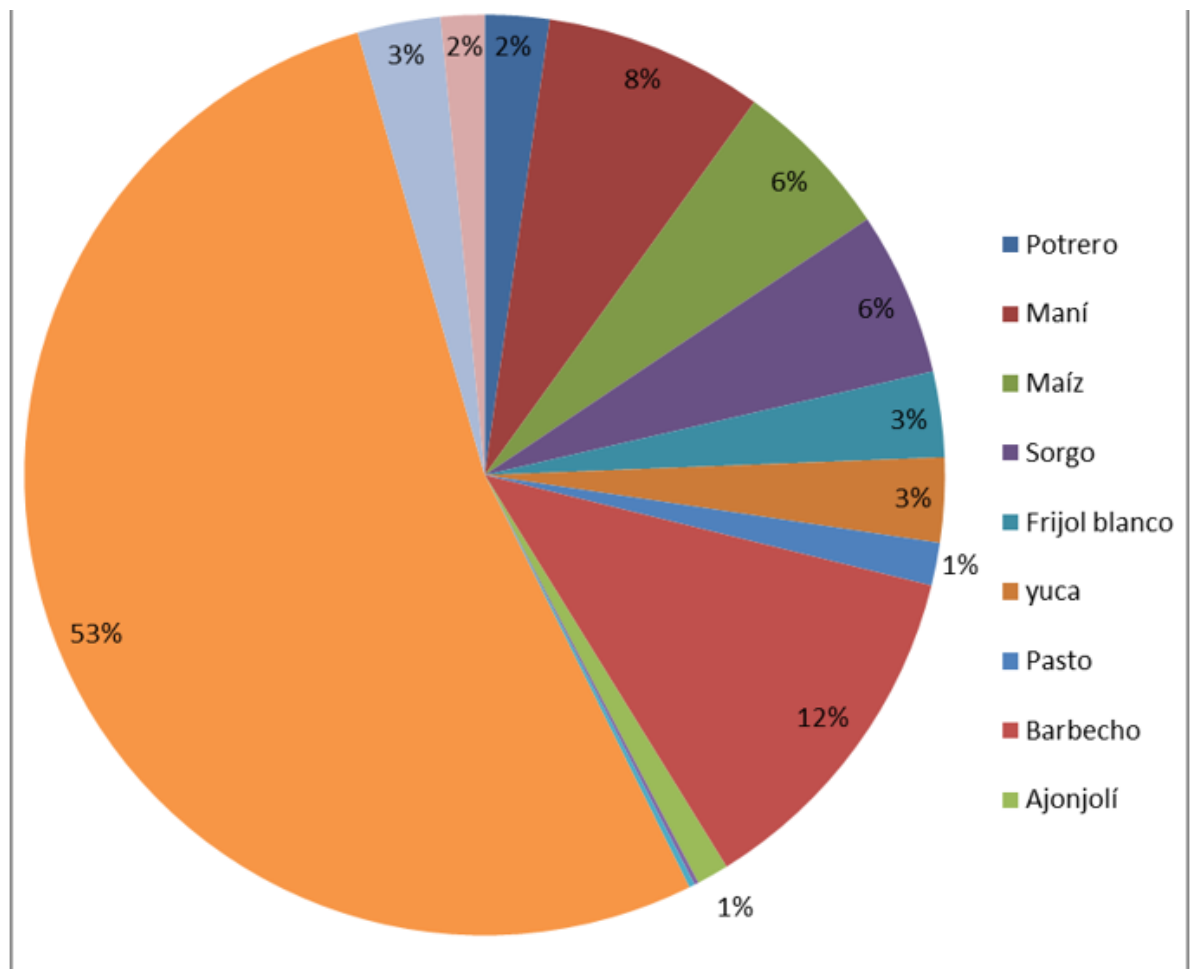

GRAFICO 21.

Distribución del uso de la tierra en todo el complejo volcánico Pilas - el Hoyo 


\subsection{Calendario floral de las especies de la flora de aprovechamiento apícola de la zona del complejo volcánico Pilas el Hoyo.}

En el complejo volcánico Pilas- El Hoyo se encontró una mayor floración entre los meses de Diciembre a Mayo, de Junio a Noviembre hay una escasez de floración existen 14 especies que florecen durante todo el año y algunos cultivos presentes en la zona lo que hace notar la importancia de estas y los cultivos para la alimentación de las abejas durante el periodo de escasez floral.

\section{Conclusiones.}

1. En el complejo volcánico Pilas- El Hoyo existe una variada oferta florística, se identificaron 89 especies, de las cuales se destacan el Sardinillo (Tecoma Stan), Eucalipto (Eucalyptus camaldulensis), Chaperno (Lonchocarpus rugosus), cinco negritos (Lantana urticifolia), Achopaste (Varronia inermis), Cornizuelo (Vachellia collinsii), Escoba amarilla (Scoparia dulcis I), escoba lisa (Waltheria indica l). Pertenecientes a 39 familias, según su estrato vegetal estas especies se dividen en: 59 especies de árboles siendo este el 66.29 \%, 15 especies de arbustos con el $16.85 \%$ y 15 especies de hierbas con el $16.85 \%$.

2. Las familias de árboles con mayor dominancia dentro de todo el complejo volcánico Pilas- el Hoyo son la Bignoniácea, Myrtaceae, Fabácea, Ebenácea y Boraginácea.

Las familias de arbustos con mayor dominancia dentro de todo el complejo volcánico Pilas- el Hoyo son la Verbenácea, Boraginaceae, Fabaceae, Apocynaceae y Solanaceae.

Las familias de hierbas con mayor dominancia dentro de todo el complejo volcánico Pilas- el Hoyo son la Plantaginacea, Malvaceae, Sterculiaceae, Lamiaceae y Convolvulacea.

3. De acuerdo a los datos de uso actual de la tierra generados del estudio:

El complejo volcánico "Pilas - el Hoyo" es una excelente zona para practicar la apicultura ya que cuenta con un 53\% de bosque silvestre de su area total, siendo el apiario la Diabla el que presenta una mayor cantidad de hectáreas con 233.26, no obstante este porcentaje de bosque silvestre se ve amenazado por el aumento de áreas cultivadas con maní la cual no solo aumenta anualmente si no que su manejo es un peligro para la subsistencia de las abejas.

4. En el complejo volcánico Pilas- El Hoyo existe una mayor floración entre los meses de Diciembre a Mayo, de Junio a Noviembre hay una escasez de floración la que se trata de compensar con algunas especies que florecen durante todo el año y algunos cultivos presentes en la zona.

\section{REcomendaciones.}

Establecer programas de reforestación con especies de aprovechamiento apícola adaptadas al clima de la zona, con monitoreo de parte $\mathrm{d}$

- Establecer programas de reforestación con especies de aprovechamiento apícola adaptadas al clima de la zona, con monitoreo de parte de la UNAN-Leon u otras organizaciones gubernamentales o no gubernamentales para asegurar la prevalencia de estas en el lugar.

- Propagar las especies que florecen todo el año, presentes en el complejo volcánico Pilas - el Hoyo, introducir nuevas especies que florezcan durante la época de escasez floral e implementar cultivos durante esta temporada para contrarrestar la misma.

- Optar por una agricultura más ecológica, haciendo uso de distintas prácticas como el manejo integrado de plagas y fertilización orgánica, para disminuir el uso de plaguicidas y fertilizantes químicos que afectan a las abejas y contaminan el ambiente. 
- Establecer un vivero con las especies más importantes presente dentro del complejo volcánico Pilas - el hoyo.

- Realizar un manejo adecuado a las plantaciones de árboles con fines energéticos como el Eucalipto, para así disminuir y prevenir el despale de las demás especies.

Continuar realizando estudios de carácter apícola para ayudar a seguir desarrollando y tecnificando este rubro.

\section{ReFERENCIAS Bibliografícas.}

Apinet-INTA. 2000. Agrobit. Recuperado de http:www.agrobit.com/infotecnica/apicultura.

Apinet - INTA (Instituto Nacional de Tecnología Agropecuaria, AR). 2000. Cultura apícola (en línea). Buenos Aires, Argentina. Disponible en http://www.culturaapicola.com.ar/apuntes/floraapicola/flora apicola.PDF.

Bazzurro, D. 1999. Flora Apícola (en línea). Disponible en http://www.culturaapicola.com.ar/apuntes/ floraapicola/126_FloraApicola.pdf

Carvallo. G,O. 2009. Especies exóticas e invasiones biológicas. (En línea) Ciencia ahora. 12(23): 15-21.

Duttmann, C. Lorenzo, D. Jiménez. M, V. 2013. La Apicultura y Factores que Influyen en Producción, Calidad, Inocuidad y Comercio de la Miel. Investigación Intersectorial de la Sanidad Apícola en el Occidente de Nicaragua. León - Nicaragua. INTA. MAG-FOR. MAPIO

Rosillo H. 2010. Diagnóstico y plan de ordenamiento territorial en el ámbito del proyecto de caficultura sostenible de alto valor para pequeños agricultores pobres, distritos de Alonso de Alvarado roque y san Martín.

Hernández-Salas, Z. T., \& Quesada-Monge, R. F. (2000 https://www.ecured.cu/Complejo_Volcánico_[EF9] Pilas_El_Hoyo

Fundación Nicaragüense para el Desarrollo Sostenible (2010). Evaluación y Redefinición del Sistema de Áreas Protegidas Cerro Negro-Pilas-El Hoyo de las Regiones Pacífico y Centro Norte de Nicaragua[EF10] . MARENA-PROTIERRA-CBA. Disponible en: http://www.renida.net.ni/renida/marena/ RENP01F981c.pdf

Laboratorio de plantas vasculares, 2006. Inventario Florístico. Argentina. Disponible en: www.plantasvasculares.uns.edu.ar/herbario/galeria/pehuen/index.html

Lúquez, F; y Valle, M. (2008). Caracterización de los Productores Apícolas en siete Comarcas del Municipio del municipio de Camoapa, Boaco, Trabajo de diplomado. Universidad Nacional Agraria, Sede Camoapa (UNA).

Mario R. Quiñónez[EF12] .2012.Programa de Investigación Apícola IPTA-FCA-UNA. Recuperado de http:www.abc.com.py/edicion-impresa/suplementos/abc-rural/floracion-de-especies-vegetales-utiles-a-laproduccion-apícola-389181.html

Meyrat \& Alain. 2016. Árboles y arbustos predominantes de Nicaragua. Managua[EF13] .

Paguaga y Soto.2010. Árboles y arbustos de la ciudad de León. Hecho en Costa Rica por la Editorial INBio. Primera edición.[EF14]

Pietronave, H. 2001. El Apicultor, Factores Climáticos que influyen en la apicultura. Disponible en http://www.inta.gov.ar/reconquista/info/documentos/comunicacion/voces_ec os/nro_23_voces_ecos/ voces_ecos_23_nota9.pdf

Pymerural. (2012). MAGFOR realiza censo apícola 2012 a nivel del país.

ROJAS M. 2002. Composición y Estructura Horizontal de un Bosque no Intervenido por la Concesión forestal Madensa-Awastigni. Puerto Cabeza. Universidad Nacional Agraria, Nicaragua. Disponible en Internet: www.agris.una.edu.ni/tesis/.

Silva, L.M.; Restrepo, S. 2012. Compendio de calendarios apícolas de Cauca, Huila y Bolívar. Bogotá, Instituto Humboldt. 52 p. 
Ángel Benito Aguilar Cabrera, et al. Caracterización florística de las especies de aprovechamiento...

Centeno Keyling y Rodríguez Norma (2016). Tesis. Análisis de la producción de miel de abeja en Nicaragua y principales limitaciones del sector apícola para la exportación a la Unión Europea, I Semestre del 2015. , UNANManagua, 2016.

Tercero Martínez.X.L, Sequeira Castillo.C.Y.2016. Estudio de Pre-Factibilidad para el establecimiento de un apiario, Finca las Delicias, Comunidad el Tule, Municipio San Miguelito, Rio San Juan. Universidad Nacional Agraria, Nicaragua. Managua.

\section{Anexos}

\section{Tabla 1}

\begin{tabular}{|c|c|c|}
\hline Wombre olenthloo & Famills & Nombre ocmún \\
\hline Prosoals yuinore & Mmosscesese & Espino Negroialgarrobo \\
\hline Moematoxpinm brasilettio. & Fasocesse. & Brasil. \\
\hline Cassia granolis & Fasocesese. & Carao \\
\hline Trebeuvis ochroces & Eignoniscese. & Cotez \\
\hline Prmecellobium ouke. & Fasocesese. & Espha DukeMlich guiste \\
\hline Somanes/Aratizia saman. & Fabacese. & Genizaro \\
\hline Lyyloma auritum & Fabocese & Quebracho \\
\hline Luenes canolbes & Nocese. & Guaciono de maienilo \\
\hline Guazuma vimitois & sterculiscesese. & Guacimo de bentero \\
\hline Aiblitia nibpables & Mimosscesese & Guanacaste bianco \\
\hline Enteralobum cyeicacspum & Mimosscesese & Guanacaste negro \\
\hline Cecroplis petrots & Cescopisceses. & Guarumo \\
\hline Prílum guagavs & Myrtasesese. & Guayasa \\
\hline Karminsksts caloeront & Fhamnaceses & nolitste \\
\hline crescentia cujese & Bivnoniscese. & Jicaro \\
\hline Bursera simarubs & Eurseracese & Jinocueso \\
\hline soonstas mombin L & Anscardiacese. & Jobo \\
\hline anceresesepum & Fasocesese. & Maserer negro \\
\hline Thounisum decanorum & sapinataces. & Metera \\
\hline Carolia collococes & Eoropinaceas. & Muneco \\
\hline Eysisonims crassitiala & Malobianiscese. & Naxite \\
\hline sepranthus wabsceus & Ammonscese. & Falanoo \\
\hline coccoboes crarcassans Nelns & Poypanoses. & Papesurso \\
\hline Cochospenmum vetrocium & Exascese. & Foroponar, berberilo \\
\hline Tabeevia rosea & Elqunoniscese. & Robie \\
\hline Tecame stans & Eivnoniscese. & Sardwilo \\
\hline Covolis dentarata & Ecraginocese. & Traylone \\
\hline semms stomensens & cossabinintereas. & Vainllo \\
\hline Eucalyotus camenoulencis & Wytacese. & Eucalpto \\
\hline Eucalyetus Uroghile & Wyrascese. & Eucalpto rajo \\
\hline colycoponytum cansolisimun & Rublesceas. & Mosrono \\
\hline Hoematioxpium brasletto & Fabocese & Brasil. \\
\hline Swletenia numils & Neliscese. & croosa \\
\hline Tectons granols $x$. & Iomiocese & тесе \\
\hline Tamarinous indica & Fabacese. & Tamarindo \\
\hline Lanchocarous nugosus & Fabacese: & Chapeno \\
\hline Carvila allowars & Boraginacese. & Lasel \\
\hline Cesersabints enostactys Benfm & Coessabiniscese & Pintadillo \\
\hline Azadivachts inates & Nelisceses. & Noem \\
\hline coessabibins veluths & Cesabioniscese & Mandaguavichapeme blanco \\
\hline Nectandra megasotamices & Louracese. & Laurel negro \\
\hline Doccles mesocsaps & Fasocesse. & oj de buey \\
\hline Coccelobs Aombunss & Paypanseces. & $n$ \\
\hline Dlosseyros saliatala. & Ebensacese. & Crocoylto \\
\hline 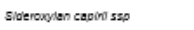 & sapoctascase. & Tempisque \\
\hline simaroubes smsera & simaroubscese. & Acetuno \\
\hline Samolas puroureo L. & Anscardiacesese. & Jocose \\
\hline Cochospentum vetronum & Exascese. & Berberilo \\
\hline Theveste anous & Aoceynsesese. & Marzano \\
\hline Feves insiobet wo d. & Noracese. & Hguera \\
\hline
\end{tabular}


TABLA 2

Especies de arbustos

\begin{tabular}{|c|c|c|}
\hline Combretum facinosum & Combretaceae. & Papamiel \\
\hline Senna pallida & Fabaceae. & Ron ren \\
\hline Kachellia collinsii & Fabaceae. & Cornizuele \\
\hline Lantana unticifolia & Verbenaceae. & Botoncillo/cinco negritos \\
\hline Stemmadenia obovata & Apocynaceae & Cachito \\
\hline Thevetia ahouai & Apocynaceae. & Manzana de jardín \\
\hline Bonellia nervosa. & Theoprhastaceae. & Cimarra \\
\hline Solanum erianthum & Solanaceae. & Lavaplatos \\
\hline Karronia inermis & Boraginaceae. & Achopaste \\
\hline Casearia corymbosa Kunth & Flacountiaceae & Frutillo \\
\hline Rhysalis peruviana & Solanaceae. & Chimbombita \\
\hline lippia cardiostegia benth & Verbenaceae. & Chichiguaste \\
\hline Malxaviscus arboreus Cax. & Malvaceae & Quesillo \\
\hline Capparis indica & Capparaceae. & Linga \\
\hline Capsicum annuum L. & Solanaceae. & Chile \\
\hline
\end{tabular}

TABLA 3

Especies de hierbas

\begin{tabular}{lll}
\hline Tetramerium nervosum Nees. & Acanthaceae. & Olotillo \\
Amaranthus spinosus L. & Amarantácea. & Bledo \\
Eclipta prostrata (L.) & Asteraceae. & Clavito \\
Alvaradoa amorphoides liebm & Picramniacea. & Cola de ardilla \\
Scoparia dulcis I & Plantaginaceae & Escoba \\
Waltheria indica I & Sterculiacea. & Escoba \\
Lantana montevidensis & Verbenaceae. & Orégano de monte \\
Amaranthus spinosus L. & Amarantácea. & Bledo amarillo \\
Justicia carthaginensis & Acanthaceae. & Flor morada \\
Hyptis suaveolens & lamiaceae. & Chan \\
Sida acuta & Malvaceaea. & Escoba blanca \\
Arachis pintoi & Fabaceae. & Maní forrajero \\
Ipomoea sp. & Convolvulaceae. & Campanita \\
Waltheria indica & Malvaceae. & Escoba pachona \\
Origanum sp & lamiaceae. & Orégano \\
\hline
\end{tabular}

